\title{
Gemeinsam lernen, miteinander teilen - Zusammenhänge zwischen dem Besuch eines inklusiven Bildungsettings und dem sozialen Verhalten von SchülerInnen ohne sonderpädagogischen Förderbedarf
}

\author{
Amelie Labsch (D) · Lena Nusser • Monja Schmitt • Marianne Schüpbach
}

Eingegangen: 12. Juli 2019 / Überarbeitet: 26. September 2020 / Angenommen: 27. Januar 2021 / Online publiziert: 18. Februar 2021

(C) Der/die Autor(en) 2021

Zusammenfassung Kinder erlernen soziales Verhalten u.a. im schulischen Kontext indem sie mit anderen SchülerInnen interagieren. Insbesondere vor dem Hintergrund der Ratifizierung der UN-Behindertenrechtskonvention stellt sich die Frage, ob sich SchülerInnen ohne sonderpädagogischen Förderbedarf (SPF) innerhalb inklusiver Klassen sozial anders verhalten als innerhalb von Klassen ohne SchülerInnen mit SPF. Mit Daten des Nationalen Bildungspanels (NEPS) zeigt sich in mehrebenanalytischer Betrachtung, dass SchülerInnen ohne SPF in inklusiven Klassen nicht von höherem prosozialem Verhalten berichten, aber von höherem Problemverhalten im Umgang mit Gleichaltrigen als ihre Peers in nicht-inklusiven Klassen. Darüber hinaus fällt ihr Problemverhalten im Umgang mit Gleichaltrigen umso höher aus, je inklusiver die Klassenkomposition gestaltet ist. Dies lässt sich über die sozioökonomische Klassenkomposition erklären, indem in inklusiven Klassen ein bedeutsam höherer Anteil an SchülerInnen mit niedrigem sozioökonomischen Status zu finden ist als in nicht-inklusiven Klassen. Die ethnische Klassenkomposition kann dagegen nicht als Erklärung herangezogen werden.

\footnotetext{
A. Labsch $(\bowtie) \cdot$ Dr. L. Nusser · Dr. M. Schmitt

Abteilung Kompetenzen, Persönlichkeit, Lernumwelten, Leibniz-Institut für Bildungsverläufe e. V., Wilhelmsplatz 3, 96047 Bamberg, Deutschland

E-Mail: amelie.labsch@lifbi.de

Dr. L. Nusser

E-Mail: lena.nusser@lifbi.de

Dr. M. Schmitt

E-Mail: monja.schmitt@lifbi.de

Prof. Dr. M. Schüpbach

Fachbereich Erziehungswissenschaft und Psychologie, Arbeitsbereich Allgemeine

Grundschulpädagogik, Freie Universität Berlin, Habelschwerdter Allee 45, 14195 Berlin,

Deutschland

E-Mail: marianne.schuepbach@fu-berlin.de
} 
Schlüsselwörter Inklusion $\cdot$ Soziales Verhalten $\cdot$ Klassenkomposition

\title{
Learning together, sharing with others-relation between attending an inclusive learning environment and the social behavior of students without special educational needs
}

\begin{abstract}
Children learn social behavior e.g. in school context by interacting with other students. In the light of the ratification of the UN Convention on the Rights of Persons with Disabilities the question arises, whether students without special educational needs (SEN) show different social behavior within inclusive classes than within classes without students with SEN. Multilevel Analyses using data of the National Educational Panel Study (NEPS) show, that students without SEN in inclusive classes don't report more prosocial behavior, but higher problem behavior with peers than those in non-inclusive classes. Moreover, the more inclusive the class composition is, the higher is their problem behavior when dealing with peers. These effects can be explained through class composition, as the percentage of students with low socioeconomic status is higher in inclusive classes than in regular classes. The ethnic class composition on the other hand can't be used as an explanation though.
\end{abstract}

Keywords Class composition · Inclusion · Social behavior

\section{Einleitung}

Beziehungen unter Gleichaltrigen entstehen, sobald sich die Gelegenheit ergibt, also wenn sie sich ,schlicht in physischer Nähe zueinander befinden“ (Zander et al. 2017, S. 367). Eine Gelegenheit hierzu wird Kindern und Jugendlichen bspw. in der Schule geboten, in der Peers formal zu Klassen und Lerngruppen zusammengeschlossen werden. Die MitschülerInnen fungieren dort als wichtigen Kontext, in dem sozialer Austausch stattfinden kann (Kiesner et al. 2003), indem sie u.a. in strukturierten Lerngelegenheiten miteinander diskutieren, an verschiedenen Inhalten zusammenarbeiten und den gegenseitigen Umgang mit Gleichaltrigen sowie Erwachsenen beobachten können (Fend 2006). Da Peers insbesondere in der Jugendphase den Prozess des Erwachsenwerdens begleiten, sind sie von zentraler Bedeutung für die individuelle Entwicklung (Hartup 2009). In Interaktionen mit Gleichaltrigen können dabei auch (unbewusst) Prozesse der Peerbeeinflussung stattfinden, indem Kinder und Jugendliche Emotionen oder z. B. soziale Verhaltensweisen ihrer Peers für sich übernehmen (für einen Überblick siehe Dishion und Tipsord 2011). Derartige Prozesse basieren auf einem sozialisatorischen Einfluss, der sich aus gewissen sozialen Unterstützungs-, Vergleichs- sowie Angleichungsmechanismen ergibt (Hartup 2009; Zander et al. 2017).

Hinsichtlich des sozialen Verhaltens von Kindern und Jugendlichen liegt der Fokus der Forschung vornehmlich darauf, Prozesse der Peerbeeinflussung über individuelle Merkmale der interagierenden SchülerInnen oder Merkmale der sozialen Umgebung zu erklären (z.B. Barry McNamara und Wentzel 2006; Hofmann und 
Müller 2018). Merkmale der sozialen Umgebung werden dabei in der Regel als Prozessmerkmale und seltener als rein strukturelle Gegebenheiten im Sinne der Komposition von Schulklassen verstanden. Dieser Forschungslücke widmet sich der Beitrag unter besonderer Berücksichtigung des gemeinsamen Lernens von SchülerInnen mit sonderpädagogischem und ohne sonderpädagogischen Förderbedarf (SPF) - hier begriffen als eine wesentliche strukturelle Komponente von Unterricht.

\section{Facetten sozialen Verhaltens}

Soziales Verhalten oder auch soziale Kompetenzen sind als Metakonstrukt mehrerer spezifischer Kompetenzen zu begreifen. Als Ausdruck sozial kompetenten Verhaltens gelten Verhaltensäußerungen in sozialen Kontexten bzw. in Beziehungen zwischen Menschen. Es umfasst sowohl den Umgang mit sich selbst, als auch mit anderen Personen und trägt wesentlich zum privaten und beruflichen Erfolg sowie individuellen Wohlbefinden bei (Kanning 2002). Entsprechende Verhaltensweisen in sozialen Beziehungen können sowohl positiv konnotiert sein, etwa durch soziale Verträglichkeit, als auch negativ, bspw. durch negative Emotionen, Konflikte oder sogar Aggressionen (Kuhn und Fischer 2011; Mahoney et al. 2003). Als eine Facette sozial kompetenten Verhaltens kann prosoziales Verhalten gefasst werden, also die Absicht einer Person, einem anderen Menschen eine Wohltat zu erweisen und dabei keinen eigenen Nutzen zu erwarten (Bierhoff 2002). Eine weitere zentrale Facette sozialen Verhaltens kann durch die Fähigkeit, positive Beziehungen und Interaktionen mit Gleichaltrigen zu führen bzw. ein geringes Problemverhalten im Umgang mit Gleichaltrigen zu zeigen, beschrieben werden (Howes 1988). Dies drückt sich bspw. in interaktiven und gemeinsamen Spielaktivitäten oder Arbeiten, emotionalem Eingehen auf soziale Andere oder Verträglichkeit mit Gleichaltrigen aus (für einen Überblick siehe Fabes et al. 2009; Howes 1988).

Bereits vielfach belegt wurde, dass das individuelle soziale Verhalten mit gewissen Personenmerkmalen assoziiert ist. Bspw. zeigen Mädchen höheres prosoziales Verhalten als Jungen (Barry McNamara und Wentzel 2006) und Beziehungen unter Jungen gehen häufiger mit antisozialem Verhalten einher als unter weiblichen Peers (Wentzel und Erdley 1993). Außerdem liegen Ergebnisse darüber vor, dass sich Kinder und Jugendliche mit Migrationshintergrund (MIG) prosozialer als diejenigen ohne einen solchen verhalten (Preuss-Lausitz 2005). Es zeigt sich auch, dass diejenigen aus bildungsfernen Elternhäusern bzw. solchen mit einem niedrigen sozioökonomischen Status aufgrund von kulturellen und kommunikativen Praktiken innerhalb der Familien über geringere Sozialkompetenzen verfügen (Szczesny und Watermann 2011).

Ein weiterer breiter Forschungsstrang widmet sich der Frage, inwiefern individuelle soziale Verhaltensweisen mit Merkmalen der sozialen Umgebung in Schulen assoziiert sind. Dabei ist zwischen Prozessen und Strukturen der Umgebung zu differenzieren. Prozessmerkmale beziehen sich auf das aktive Geschehen zwischen den beteiligten Akteuren etwa bei der Vermittlung von Lerninhalten. So zeigt sich bspw., dass das schulische Problemverhalten auf Klassenebene weniger die Entwicklung des individuellen schulischen Problemverhaltens beeinflusst, sobald die Anleitung 
durch Lehrkräfte als unterstützend oder interessant empfunden wird (Müller et al. 2018). Auch durch die Einbindung der SchülerInnen in die Unterrichtsgestaltung und die Anregung zu Kooperation im Unterricht durch die Lehrkraft kann das soziale Verhalten begünstigt werden (Battistich et al. 1997). Strukturmerkmale hingegen gelten eher als kompositionelle Gegebenheiten, die sich aus der Zusammensetzung von SchülerInnen mit den unterschiedlichsten individuellen Merkmalen in Klassen ergeben. Längsschnittuntersuchungen zeigen, dass ein hoher Aggressionslevel auf Klassenebene mit einem hohen individuellen Aggressionsverhalten einhergeht (Müller et al. 2015). Zwar kann dieser Zusammenhang durch strukturierte Interventionen zum Aggressionsverhalten im Schulkontext abgeschwächt werden, aber dennoch bleibt eine Langzeitwirkung des Aggressionsverhaltens auf Klassenebene auf das spätere individuelle aggressive Verhalten bestehen (Kellam et al. 1998). Auch eine hohe durchschnittliche Prosozialität der MitschülerInnen einer Klasse verringert das individuelle antisoziale Verhalten nachhaltig (Hofmann und Müller 2018). Außerdem finden Demanet und van Houtte (2011) in einer Befragung von Dritt- und FünftklässlerInnen negative Zusammenhänge zwischen einer Schulkomposition mit niedrigem soziö̈konomischen Status und dem schulischen Problemverhalten von SchülerInnen, was laut der AutorInnen auf die besuchte Schulform zurückzuführen ist.

Zusammenfassend bedeuten die dargestellten Befunde, dass durch die Zuordnung von SchülerInnen zu Klassen bzw. Lerngruppen ein struktureller Kontext des Lernens vorgegeben wird, in dem sich sowohl individuelle Merkmale von SchülerInnen als auch Verhaltensweisen von MitschülerInnen bzw. deren aggregierte Merkmale auf Klassenebene auf das individuelle soziale Verhalten auswirken können.

\section{Inklusives Bildungssetting als Kontextmerkmal}

In den letzten Jahren hat die Entwicklung von SchülerInnen in zunehmend heterogenen Settings für die Forschung und Praxis an Bedeutung gewonnen. Dies ist unter anderem mit der Ratifizierung der UN-Behindertenrechtskonvention verbunden, mit der sich Deutschland 2009 zu einem inklusiven Schulsystem verpflichtet hat. Als Konsequenz besuchen SchülerInnen mit und ohne SPF vermehrt gemeinsam allgemeine Schulen (Tenorth 2013). Das bedeutet auch, dass durch die Platzierung der SchülerInnen mit SPF im Regelschulsystem die bereits vorhandene Vielfalt der SchülerInnen innerhalb eines Klassenkontextes um ein weiteres kompositionelles Merkmal der sozialen Umgebung von SchülerInnen ergänzt wird. Entsprechend sind Prozesse der Peerbeeinflussung zwischen SchülerInnen mit den unterschiedlichsten kognitiven, physischen und psychischen Merkmalen, Voraussetzungen und Verhaltensweisen von Interesse. Daher sollte hinsichtlich des sozialen Verhaltens von SchülerInnen neben den oben dargestellten Merkmalen und Verhaltensweisen der MitschülerInnen auch das inklusive Bildungssetting als Kontextmerkmal der Klassenkomposition berücksichtigt werden.

Wie sich das soziale Verhalten von SchülerInnen in inklusiven Bildungssettings entwickelt, wird bislang eher mit vergleichendem Blick auf SchülerInnen mit und ohne SPF innerhalb inklusiver Klassen (Frostad und Pijl 2007; Hennig et al. 2017) 
oder an Regel- und Förderschulen untersucht. Dabei zeigt sich bspw., dass SchülerInnen mit Förderbedarf im Bereich Lernen an Förder- und Regelschulen häufiger als SchülerInnen ohne entsprechenden Förderbedarf von höherem Problemverhalten im Umgang mit Gleichaltrigen berichten (Schwab et al. 2016). Dass SchülerInnen mit SPF für diejenigen ohne einen solchen hinsichtlich sozialer Verhaltensweisen ebenfalls als Kontext für mögliche Peereinflussprozesse fungieren können, wird dabei vernachlässigt. Gerade bei SchülerInnen mit dem Förderschwerpunkt emotionale und soziale Entwicklung sind Auffälligkeiten im Verhalten und Erleben erkennbar, indem diese häufig unsozial, impulsiv und teilweise aggressiv sind (Stein und Müller 2018). Bei SchülerInnen mit SPF im Bereich Lernen sind häufig kognitive Auffälligkeiten, deutliche inhaltliche Lernrückstände sowie eine geringe Konzentrationsfähigkeit auszumachen (Grünke und Grosche 2014). Weiter zeigt auch diese Gruppe häufig externalisierendes Problemverhalten, also u. a. aggressives oder delinquentes Verhalten, sowie soziale Probleme im Umgang mit Gleichaltrigen (Fischbach et al. 2010). Ergebnisse aus einer wiederholten Befragung im Primarschulbereich zeigen, dass SchülerInnen ohne SPF mehr externalisierendes und internalisierendes Verhalten sowie weniger Selbstkontrolle und soziale Fähigkeiten zeigen, insbesondere dann, wenn mehr als ein/e SchülerIn mit SPF in der Klasse anwesend ist (Gottfried 2014). Diesen Befund erklärt Gottfried (2014) mit einem negativen Peereffekt, indem sich SchülerInnen ohne SPF an das Sozialverhalten ihrer MitschülerInnen mit SPF angleichen. Zudem führt er die Effekte auf den Förderbedarf der SchülerInnen mit SPF zurück und zeigt bspw., dass SchülerInnen mit Förderbedarf im Bereich „emotional/behavioral disorders“ am stärksten das Verhalten ihrer MitschülerInnen bedingen. Dies geht auch mit Ergebnissen einher, dass SchülerInnen mit Förderbedarf im Bereich Verhalten weniger Empathie zeigen, was gleichzeitig mit einer geringeren sozialen Position einhergeht (Frostad und Pijl 2007). Die AutorInnen der Studie begründen damit einen Mangel an Lerngelegenheiten für soziale Kompetenzen. In einer querschnittlichen Untersuchung im Primarbereich wird außerdem deutlich, dass SchülerInnen mit SPF häufiger aus Familien mit einem eher niedrigen sozioökonomischen Status (SES) kommen (Henke et al. 2017).

Die dargestellten Befunden zeigen, dass eine Schulkomposition mit niedrigem sozioökonomischen Status schulisches Problemverhalten verstärken kann (Demanet und van Houtte 2011). Weiter kommen, SchülerInnen mit SPF häufig aus Familien mit niedrigem SES (Henke et al. 2017) und zeigen gleichzeitig höheres Problemverhalten im Umgang mit Gleichaltrigen (Schwab et al. 2016). Zudem können sich negative Peereffekte für SchülerInnen ohne SPF in einer inklusiven Lernumgebung ergeben (Gottfried 2014). Entsprechend lässt sich ableiten, dass SchülerInnen mit SPF als Rollenmodell für SchülerInnen ohne SPF fungieren und dass diese entsprechende Verhaltensweisen übernehmen und ungünstiges soziales Verhalten zeigen könnten. Neben gewissen Angleichungseffekten ist außerdem zu beobachten, dass SchülerInnen ohne SPF in inklusiven Klassen häufig Vorurteile gegenüber ihren MitschülerInnen mit SPF haben, sie als ,anders“, hilflos und mitleidsbedürftig wahrnehmen (Hazzard 1983). Entsprechend ist auch anzunehmen, dass sich besonders in inklusiven Settings weniger intensive Beziehungen unter Peers ergeben, was sich wiederum auf den Umgang mit Gleichaltrigen auswirken könnte. 


\section{Forschungsfrage}

Wenn auch SchülerInnen ohne SPF bspw. hinsichtlich ihrer akademischen Entwicklung von dem Besuch inklusiver Settings profitieren können (für einen Überblick siehe Ruijs und Peetsma 2009), vermittelt der dargestellte Forschungsstand ein eher negatives Bild bezüglich ihres sozialen Verhaltens. Allerdings fanden die Untersuchungen zum sozialen Verhalten von SchülerInnen ohne SPF in inklusiven Settings häufig im Primarschulbereich statt und zudem wurden nur selten verschiedene Merkmale der Klassenkomposition berücksichtigt. Der Fokus lag vornehmlich auf dem strukturellen Merkmal der Anwesenheit von SchülerInnen mit SPF, während bereits bestehende Merkmale der Klassenkomposition und der Individuen in inklusiven Settings nicht betrachtet wurden. Entsprechend wird dabei vernachlässigt, dass Bildungssettings, ehe sie durch die Aufnahme von SchülerInnen mit SPF inklusiv werden, bereits eine bestimmte sozioökonomische und ethnische Klassenkomposition aufweisen, die durch die Anwesenheit von SchülerInnen mit SPF um ein weiteres Merkmal ergänzt wird.

Folglich soll der vorliegende Beitrag die Frage beantworten, ob vor dem Hintergrund bestimmter bestehender Kompositionsmerkmale der Klassen und Merkmale der Individuen Zusammenhänge zwischen dem prosozialen Verhalten sowie dem Problemverhalten im Umgang mit Gleichaltrigen von SchülerInnen ohne SPF und dem Besuch eines inklusiven Settings bestehen.

Aufgrund der dargestellten Befunde wird angenommen, dass ein statistisch bedeutsamer Unterschied im sozialen Verhalten von SchülerInnen ohne SPF in Klassen, in denen SchülerInnen mit SPF anwesend sind (inklusive Klassen) und solchen, in denen keine SchülerInnen mit SPF anwesend sind (nicht-inklusive Klassen), vorliegt. Es wird erwartet, dass SchülerInnen ohne SPF generell weniger prosoziales (H1a) und höheres Problemverhalten im Umgang mit Gleichaltrigen zeigen als ihre Peers in nicht-inklusiven Klassen (H1b).

Außerdem wird davon ausgegangen, dass SchülerInnen ohne SPF in Klassen mit einer zunehmend inklusiven Klassenkomposition, also einem steigenden prozentualen Anteil an anwesenden SchülerInnen mit SPF, mehr Lerngelegenheiten für ungünstige Verhaltensweisen haben. Dies führt dazu, dass SchülerInnen ohne SPF in inklusiven Klassen auch bei einer zunehmend inklusiven Klassenkomposition statistisch signifikant geringeres prosoziales Verhalten (H2a) und bedeutsam höheres Problemverhalten im Umgang mit Gleichaltrigen zeigen als diejenigen in nicht-inklusiven Klassen (H2b).

Obwohl Befunde zeigen, dass sich eine Klassenkomposition mit niedrigem sozioökonomischen Status negativ auf das soziale Verhalten auswirken kann (Demanet und van Houtte 2011) und SchülerInnen mit SPF häufig einen niedrigeren SES aufweisen (Henke et al. 2017), wird angenommen, dass das inklusive Kompositionsmerkmal als zusätzliches Strukturmerkmal mit dem sozialen Verhalten von SchülerInnen ohne SPF assoziiert ist. Es wird erwartet, dass die negative Beziehung bestehen bleibt, wenn für die sozioökonomische (H3a) und ethnische (H3b) Klassenkomposition kontrolliert wird. Auch für das Problemverhalten im Umgang mit Gleichaltrigen wird angenommen, dass dieses eher mit Merkmalen der Unterschiedlichkeit im Verhalten und Erleben als mit Personenmerkmalen wie dem MIG 
zusammenhängt und sich folglich die negativen Zusammenhänge auch dann zeigen, wenn für die sozioökonomische $(\mathrm{H} 3 \mathrm{c})$ und ethnische (H3d) Klassenkomposition kontrolliert wird.

\section{Methode}

\subsection{Stichprobe}

Zur Analyse der Forschungsfrage werden Daten des Nationalen Bildungspanel (Blossfeld und Roßbach 2011) ${ }^{1}$ aus der Startkohorte 3 herangezogen. Insgesamt nahmen an der ersten papierbasierten Erhebung im Schulkontext in der fünften Jahrgangsstufe $N=5753$ SchülerInnen freiwillig teil. Diese wurden in ihrem Bildungsweg durch die Sekundarstufe I begleitet und in der Regel einmal pro Schuljahr wiederholt befragt. Um Hintergrundmerkale der SchülerInnen und Kontextmerkmale der Bildungssettings zu erfassen, wurden u. a. ihre Eltern telefonisch und die unterrichtenden Klassenlehrkräfte schriftlich um Angaben gebeten.

Aus den Analysen wurden diejenigen SchülerInnen ausgeschlossen, die nach Angaben ihrer Lehrkräfte eine diagnostizierten SPF aufwiesen ${ }^{2}(n=124)$ oder deren Lehrkräfte keine Angabe zur Anwesenheit von SchülerInnen mit SPF in den Klassen machten. Auch SchülerInnen, die Förderschulen oder ein sechsjähriges Grundschulsystem besuchten, wurden für die Analysen nicht berücksichtigt. In der Stichprobe befinden sich daher nur SchülerInnen ohne SPF, die eindeutig über die Angabe der Lehrkräfte einer inklusiven oder nicht-inklusiven Klasse zugeordnet werden konnten. Da die darüber hinaus bestehenden Missings in den relevanten Variablen nicht auf einen systematischen Ausfall hinweisen, gingen nur Fälle ohne fehlende Werte in die Analyse ein.

Letztlich konnten Daten von $n=1563$ SchülerInnen ohne SPF in die Analysen aufgenommen werden, von denen etwa $20 \%$ inklusive Klassen besuchten. An der NEPS-Erhebung nahmen pro betrachteter Klasse durchschnittlich fünf SchülerInnen ohne SPF teil.

\footnotetext{
${ }^{1}$ Diese Arbeit nutzt Daten des Nationalen Bildungspanels (NEPS): Startkohorte Klasse 5, https://doi.org/ 10.5157/NEPS:SC3:7.0.1. Die Daten des NEPS wurden von 2008 bis 2013 als Teil des Rahmenprogramms zur Förderung der empirischen Bildungsforschung erhoben, welches vom Bundesministerium für Bildung und Forschung (BMBF) finanziert wurde. Seit 2014 wird NEPS vom Leibniz-Institut für Bildungsverläufe e.V. (LIfBi) an der Otto-Friedrich-Universität Bamberg in Kooperation mit einem deutschlandweiten Netzwerk weitergeführt.

2 Zum Zeitpunkt der Erhebung (2010) bildeten SchülerInnen mit den Förderschwerpunkten „emotionale und soziale Entwicklung“ (23,5\%) sowie „Lernen“ (43,5\%) die größte Population unter den inklusiv lernenden SchülerInnen mit SPF in Deutschland (Sekretariat der Ständigen Konferenz der Kultusminister der Länder in der Bundesrepublik Deutschland 2016).
} 


\subsection{Prosoziales Verhalten und Problemverhalten im Umgang mit Gleichaltrigen}

Das prosoziale Verhalten und Problemverhalten im Umgang mit Gleichaltrigen wurde in der zweiten Befragung in der sechsten Jahrgangsstufe mit dem Strengths and Difficulties Questionnaire (SDQ; Goodman 1997) erhoben. Dieser misst über verschiedene Dimensionen Stärken und Schwächen von Kindern und Jugendlichen im Alter von vier bis 16 Jahren. In der Erhebung wurden die Dimensionen prosoziales Verhalten und Problemverhalten im Umgang mit Gleichaltrigen eingesetzt. Dabei ist letztere Dimension eine von vier Problemskalen des SDQ, während das prosoziale Verhalten als Stärke definiert wird (Goodman 1997). Mit Hilfe der Version zur Selbsteinschätzung wurden die SchülerInnen über jeweils fünf Items gebeten, Aussagen über ihr prosoziales Verhalten (z. B.: „Ich helfe anderen oft freiwillig.“) und ihr Problemverhalten im Umgang mit Gleichaltrigen (z. B. „Ich komme besser mit Erwachsenen aus als mit Gleichaltrigen.") zu bewerten. Die abhängigen Variablen wurden jeweils auf einer dreistufigen Skala $(0=$,nicht zutreffend“ $1=$, ,teilweise zutreffend“", 2 = „eindeutig zutreffend“) abgebildet und anschließend zu jeweils einem Summenscore im Bereich von 0-10 zusammengefasst. Sofern in den Items Missings vorlagen, wurden die Fälle aus der Summenscore-Berechnung ausgeschlossen. Dabei gilt, je höher die Summe, desto höher bewerteten die SchülerInnen ihr prosoziales Verhalten bzw. desto höher schätzten sie ihr Problemverhalten im Umgang mit Gleichaltrigen ein. Die interne Konsistenz der Skala prosoziales Verhalten weist für die Stichprobe der Untersuchung einen zufriedenstellenden Wert auf $(\alpha=0,71)$. Das Problemverhalten im Umgang mit Gleichaltrigen hingegen erzielt für die Gesamtstichprobe der NEPS-Erhebung ein Cronbachs alpha von $\alpha=0,82$; für die vorliegende Stichprobe allerdings einen mangelhaften Wert von $\alpha=0,59$. Auch in anderen Studien finden sich insbesondere für diese Dimension des SDQ geringe Werte der internen Konsistenz. Lohbeck et al. (2015) begründen dies bspw. mit der geringen Anzahl an Items, mit denen ein sehr breiter Merkmalsbereich abgefragt wird und bewerten dies für ein Screening-Instrument wie es der SDQ ist als durchaus ,wünschenswert", weil so festgestellt werden kann, dass die Skalen durchaus unterschiedliche Facetten von Stärken und Schwächen ansprechen. Mit letzter Argumentation geht einher, dass die beiden Skalen schwach negativ untereinander korrelieren, weshalb sie als getrennt zu betrachtende Facetten sozialen Verhaltens untersucht werden $(r=-0,15)$.

\subsection{Unabhängige Variablen}

Merkmale der Klassenkomposition Um festzustellen, ob die SchülerInnen inklusive Klassen besuchten, wurde die Information der Klassenlehrkräfte genutzt, ob zum Befragungszeitpunkt SchülerInnen mit SPF in ihren Klassen anwesend waren. Diese gaben in einer halboffenen Frage an, wie viele SchülerInnen mit einem diagnostizierten SPF in der Klasse unterrichtet werden. An der NEPS-Erhebung in allgemeinen Schulen selbst nahmen SchülerInnen mit Förderbedarfen u. a. in den Bereichen Lernen, Sprache, emotionale und soziale Entwicklung und Autismus teil, wobei SchülerInnen des ersten Bereichs die größte Gruppe bilden (ca. 30\%). Aufgrund der geringen Fallzahlen für die einzelnen Förderbereiche der SchülerInnen 
mit SPF wurde in den Analysen nicht weiter nach verschiedenen Gruppen differenziert und folglich die sehr heterogene Gruppe zusammengefasst. Entsprechend gilt für die vorliegende Darstellung eine inklusive Klasse als strukturelles Merkmal, dass mindestens ein/e SchülerIn mit SPF unabhängig von der Art des Förderbedarfs in der Klasse anwesend ist. Diese Herangehensweise erlaubt, in den Analysen einerseits mit einer Dummy-Variable zwischen inklusiver und nicht-inklusiver Klasse $\mathrm{zu}$ unterscheiden und andererseits die inklusive Klassenkomposition bezüglich der Zusammensetzung aus SchülerInnen mit und ohne SPF innerhalb der Klassen in die Analysen aufzunehmen (prozentualer Anteil an SchülerInnen mit SPF in den Klassen: $M=1,75 ; S D=4,75 ; M i n=0 ; M a x=40)$. Auch für die Information zur sozioökonomischen und ethnischen Klassenkomposition wurde auf Aussagen der Klassenlehrkräfte über den gesamten Klassenkontext zurückgegriffen, da die Elternangabe zum SES und MIG der Familie nur für SchülerInnen vorliegt, die an der NEPS-Erhebung teilgenommen haben. Diese wurden gefragt, wie viele Kinder mit niedrigem SES und MIG in ihren Klassen anwesend waren. MIG wird hier so verstanden, dass mindestens ein Elternteil der SchülerInnen im Ausland geboren wurde. Als Kompositionsmerkmal gilt jeweils der prozentuale Anteil an SchülerInnen mit den entsprechenden Merkmalen einer Klasse im Verhältnis zur Klassengröße.

Die Angaben zur Klassenkomposition stammen aus der vorangegangen Erhebungswelle in der fünften Jahrgangsstufe, da die Erhebung in der sechsten Jahrgangsstufe bereits zu Beginn des Schuljahres erfolgte. Somit kann gewährleistet werden, dass in den Klassen über mindestens ein Jahr SchülerInnen mit und ohne SPF gemeinsam unterrichtet wurden oder dies eben genau ausgeschlossen ist.

Individualmerkmale Geschlecht, SES (HISEI: Highest International Socio-Economic Index of Occupational Status; Ganzeboom et al. 1992; Ganzeboom und Treiman 1996), sowie $M I G$ der SchülerInnen wurden als Kontrollvariablen aufgenommen. Die Information zum SES und MIG als Individualmerkmal stammen aus dem telefonischen Interview mit den Eltern. Auch hier wurden SchülerInnen dann als MigrantInnen definiert, wenn mindestens ein Elternteil im Ausland geboren wurde.

\subsection{Analytisches Vorgehen}

Sämtliche Analysen wurden mit dem Statistikprogramm Stata 15 berechnet. Zunächst werden Deskriptiva betrachtet. Da die SchülerInnen, die in die Stichprobe eingingen, in Klassen geclustert sind, erfolgte die multivariate Analyse mehrebenenanalytisch. Mit diesem Verfahren kann festgestellt werden, ob mögliche Unterschiede in den abhängigen Variablen auf der Zugehörigkeit zu inklusiven oder nichtinklusiven Klassen beruhen.

Um zu überprüfen, ob die in Hypothese 1a und $1 \mathrm{~b}$ angenommenen generellen Unterschiede zwischen den Klassensettings besteht, wurde mit Hilfe der DummyVariable zur inklusiven Klasse auf Level 2 geprüft, ob der Zusammenhang zwischen dem Besuch einer inklusiven Klasse und den abhängigen Variablen signifikant ist. Um festzustellen, ob dieser auf die inklusive Klassenkomposition (H2a, H2b) zurückzuführen ist, wurde anschließend die Angabe der Klassenlehrkräfte zum Anteil von SchülerInnen mit SPF in ihren Klassen als metrische Variable auf Level 2 in 
die Analyse aufgenommen. Auch Merkmale der sozioökonomischen und ethnischen Klassenkomposition wurden daraufhin als metrische Variablen auf Level 2 in die Modelle einbezogen, um zu untersuchen, ob Zusammenhänge zwischen einer inklusiven Klasse und den abhängigen Variablen bestehen bleiben, wenn für weitere Merkmale der Klassenkomposition kontrolliert wird (H3a-H3d).

Da der Forschungsschwerpunkt auf Zusammenhängen zwischen Merkmalen des Lernkontextes und dem sozialen Verhalten liegt, gehen individuelle Hintergrundmerkmale der SchülerInnen ohne SPF auf Level 1 als Kontrollvariablen in die Modelle ein. Die (quasi-)metrischen Variablen HISEI sowie die der inklusiven, sozioökonomischen und ethnischen Klassenkomposition wurden z-standardisiert.

\section{Ergebnisse}

Deskriptiva Wie in Tab. 1 dargestellt, schätzen SchülerInnen ohne SPF ihr prosoziales Verhalten tendenziell hoch ein $(M=7,38 ; S D=1,92)$ und berichten von einem geringen Problemverhalten im Umgang mit Gleichaltrigen $(M=2,27 ; S D=1,83)$. Bei einem t-test für unabhängige Stichproben zeigen sich keine Unterschiede im prosozialen Verhalten von SchülerInnen ohne SPF in inklusiven und nicht-inklusiven Klassen $(\mathrm{t}(1561)=1,22 ; p>0,05)$, wonach erwartbar ist, dass der inklusive Kontext nicht per se mit dem prosozialen Verhalten der SchülerInnen ohne SPF assoziiert ist. Bezüglich des Problemverhaltens im Umgang mit Gleichaltrigen sind allerdings statistisch bedeutsame Unterschiede zwischen den Gruppen erkennbar, wonach SchülerInnen ohne SPF in inklusiven Klassen signifikant höheres Problemverhalten im Umgang mit Gleichaltrigen empfinden als ihre Peers in nicht-inklusiven Klassen $(\mathrm{t}(1561)=-3,89 ; p<0,05)$. Im Vergleich der Klassenstruktur fällt außerdem auf, dass in inklusiven Klassen eine homogenere ethnische Klassenkomposition herrscht als in nicht-inklusiven Klassen, also ein signifikant geringerer Anteil Kinder mit MIG anwesend ist ( $\mathrm{t}(1451)=2,64 ; p<0,05)$. Demgegenüber weist die Klassenkomposition in inklusiven Klassen einen bedeutsam niedrigeren sozioökonomischen Status auf, wonach sich dort ein höherer Anteil an Kindern mit einem niedrigen SES befindet (t $(749)=-3,81 ; p<0,05)$. Die Betrachtung der Stichprobe bzw. der Gruppen deutet darauf hin, dass ein genereller Unterschied in den abhängigen Variablen je nach Besuch einer inklusiven oder nicht-inklusiven Klasse besteht, der möglicherweise mit der Zusammensetzung der Klassen hinsichtlich dieser Merkmale assoziiert ist.

Auch die bivariaten Korrelationen, die in Tab. 2 dargestellt sind, weisen auf einen Zusammenhang zwischen der sozioökonomischen Klassenkomposition und dem prosozialen Verhalten $(r=-0,07)$ sowie dem Problemverhalten im Umgang mit Gleichaltrigen $(r=0,16)$ hin. Der Besuch einer inklusiven Klasse $(r=0,10)$ sowie die inklusive Klassenkomposition $(r=0,11)$ sind zunächst nur mit dem Problemverhalten im Umgang mit Gleichaltrigen assoziiert.

Multivariate Analysen Durch den Klassenkontext können 3\% der Varianz des prosozialen Verhaltens und $4 \%$ der Varianz des Problemverhaltens im Umgang mit Gleichaltrigen aufgeklärt werden. Besonders im schulischen Bereich ist häufig mit einer geringen Varianzaufklärung durch den Kontext zu rechnen, weshalb es sich 


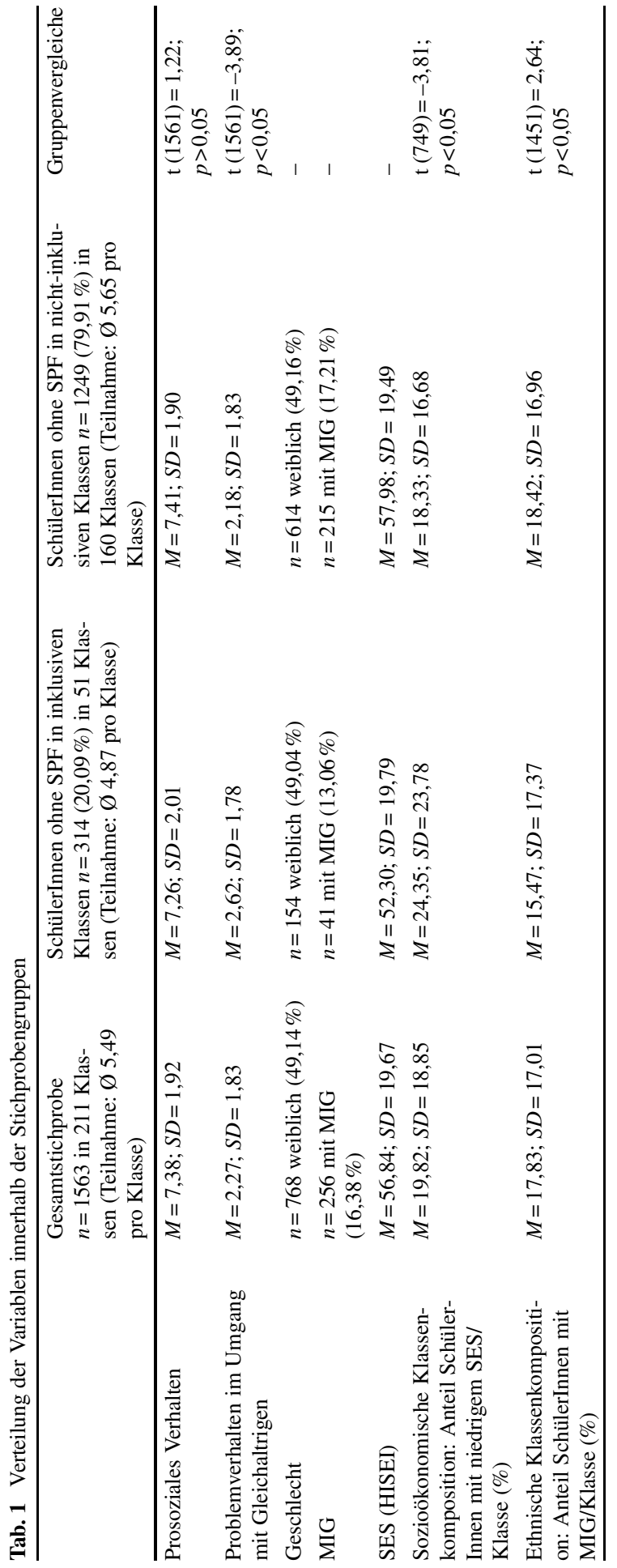


Tab. 2 Bivariate Korrelationen

\begin{tabular}{|c|c|c|c|c|c|c|c|c|c|}
\hline- & (1) & (2) & (3) & (4) & (5) & (6) & (7) & (8) & (9) \\
\hline Prosoziales Verhalten (1) & - & - & - & - & - & - & - & - & - \\
\hline $\begin{array}{l}\text { Problemverhalten im Um- } \\
\text { gang mit Gleichaltrigen } \\
\text { (2) }\end{array}$ & $-0,15^{*}$ & - & - & - & - & - & - & - & - \\
\hline Geschlecht (3) & $0,29 *$ & $-0,09^{*}$ & - & - & - & - & - & - & - \\
\hline HISEI (4) & 0,05 & $-0,10^{*}$ & $-0,02$ & - & - & - & - & - & - \\
\hline MIG (5) & 0,02 & 0,01 & 0,03 & $-0,12^{*}$ & - & - & - & - & - \\
\hline $\begin{array}{l}\text { Besuch einer inklusiven } \\
\text { Klasse (6) }\end{array}$ & $-0,03$ & $0,10^{*}$ & $-0,01$ & $-0,12^{*}$ & $-0,05$ & - & - & - & - \\
\hline $\begin{array}{l}\text { Inklusive Klassenkomposi- } \\
\text { tion (7) }\end{array}$ & $-0,02$ & $0,11^{*}$ & $-0,03$ & $-0,10^{*}$ & $-0,06^{*}$ & $0,73^{*}$ & - & - & - \\
\hline $\begin{array}{l}\text { Sozioökonomische Klassen- } \\
\text { komposition (8) }\end{array}$ & $-0,07^{*}$ & $0,16^{*}$ & $-0,08^{*}$ & $-0,27^{*}$ & $0,15^{*}$ & $0,14^{*}$ & $0,33^{*}$ & - & - \\
\hline $\begin{array}{l}\text { Ethnische Klassenkomposi- } \\
\text { tion (9) }\end{array}$ & 0,01 & 0,01 & $-0,01$ & $-0,14^{*}$ & $0,34^{*}$ & $-0,07^{*}$ & $-0,04$ & $0,54^{*}$ & - \\
\hline
\end{tabular}

$* p<0,05$

bereits bei einem geringen ICC lohnt, Mehrebenenmodelle zu spezifizieren (Hox 2010).

Wie in Tab. 3 dargestellt, zeigt sich kein statistisch bedeutsamer Unterschied zwischen dem prosozialen Verhalten von SchülerInnen ohne SPF in inklusiven und nicht-inklusiven Klassen. Folglich schätzen SchülerInnen ohne SPF ihr prosoziales Verhalten nicht geringer ein, wenn sie eine inklusive Klasse besuchen (M1: $b=-0,14$; $p>0,05)$. Da kein bedeutsamer Zusammenhang zwischen dem prosozialen Verhalten von SchülerInnen ohne SPF und dem Besuch einer inklusiven Klasse besteht, muss Hypothese 1a abgelehnt werden. Wie sich allerdings bereits in der deskriptiven Darstellung zeigte, ist das Problemverhalten im Umgang mit Gleichaltrigen signifikant positiv mit dem Besuch einer inklusiven Klasse assoziiert. Aufgrund der Skalenausrichtung bedeutet dies, dass SchülerInnen ohne SPF ihr Problemverhalten im Umgang mit Gleichaltrigen als höher bewerten, wenn sie inklusive Klassen besuchen (M7: $b=0,42 ; p<0,05)$. Weil ein generell höheres Problemverhalten im Umgang mit Gleichaltrigen in inklusiven Klassen vorliegt, kann Hypothese $1 \mathrm{~b}$ bestätigt werden.

Um die in Hypothese 2a und $2 \mathrm{~b}$ angenommenen Zusammenhänge zwischen den abhängigen Variablen und der inklusiven Klassenkomposition zu betrachten, wurden anstatt der Dummy-Variable die metrische Variable zur inklusiven Klassenkomposition in die Analysen aufgenommen. Auch hier besteht kein signifikanter Zusammenhang zwischen der inklusiven Klassenkomposition und dem prosozialen Verhalten. SchülerInnen ohne SPF berichten also auch nicht von geringerem prosozialen Verhalten, je höher der Anteil an SchülerInnen mit SPF in den Klassen ist (M2: $\mathrm{b}=-0,01 ; p>0,05)$. Es ist lediglich eine negative Tendenz erkennbar und Hypothese 2a muss abgelehnt werden. Demgegenüber besteht eine positiv signifikante Beziehung zwischen der inklusiven Klassenkomposition und dem Problemverhalten im Umgang mit Gleichaltrigen. SchülerInnen schätzen also auch dann ihr 


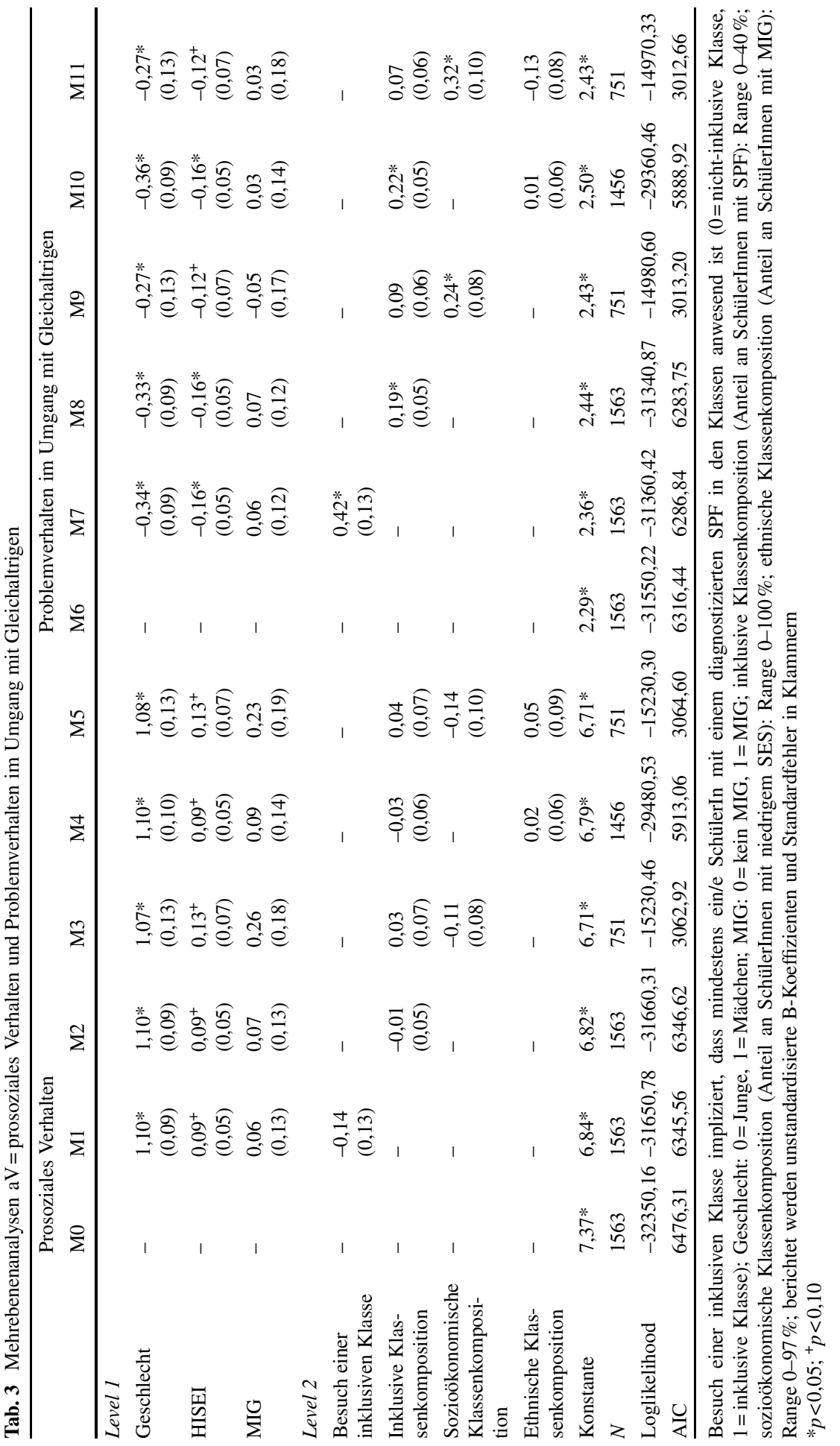


Problemverhalten im Umgang mit Gleichaltrigen höher ein, je inklusiver die Klassenkomposition hinsichtlich der Zusammensetzung von SchülerInnen mit und ohne SPF ausfällt ist (M8: $\mathrm{b}=0,19 ; p<0,05)$. Entsprechend kann Hypothese $2 \mathrm{~b}$ bestätigt werden.

Um sicherzustellen, dass die bereits betrachteten Zusammenhänge nicht auf weitere Merkmale der Klassenkomposition zurückzuführen sind, sondern es sich um unabhängige Befunde handelt, wurden im nächsten Schritt die sozioökonomische und ethnische Klassenkomposition in die Analysen einbezogen (H3a-H3d). Unter separater Hinzunahme der sozioökonomischen (M3) und ethnischen (M4) Klassenkomposition sind nach wie vor keine statistisch bedeutsamen Zusammenhänge zwischen der inklusiven Klassenkomposition und dem prosozialen Verhalten erkennbar (M3: $b=-0,03 ; p>0,05 ; M 4: b=-0,03 ; p>0,05)$. Dies lässt sich auch unter simultaner Berücksichtigung der sozioökonomischen und ethnischen Klassenkomposition replizieren (M5: $\mathrm{b}=-0,04 ; p>0,05)$. Da SchülerInnen ohne SPF weder geringeres prosoziales Verhalten zeigen, wenn neben der inklusiven Klassenkomposition die sozioökonomische (H3a) noch die ethnische (H3b) Klassenkomposition kontrolliert wird, müssen die entsprechenden Hypothesen abgelehnt werden. Für das Problemverhalten im Umgang mit Gleichaltrigen hingegen zeigt sich, dass der positive $\mathrm{Zu}-$ sammenhang mit der inklusiven Klassenkomposition durch die sozioökonomische Klassenkomposition erklärt werden kann. Folglich empfinden SchülerInnen ohne SPF ihr Problemverhalten im Umgang mit Gleichaltrigen nicht als höher, je inklusiver die Klassenkomposition hinsichtlich der Zusammensetzung von SchülerInnen mit und ohne SPF ist, sondern bei steigendem Anteil an SchülerInnen mit niedrigem SES in den Klassen (M9: $\mathrm{b}=0,09 ; p>0,05)$. Dies stimmt mit den ersten Hinweisen aus der Stichprobenbeschreibung überein. Unter Einbezug der ethnischen Klassenkomposition hingegen bleibt die positiv signifikante Beziehung bestehen, was bedeutet, dass SchülerInnen ohne SPF auch dann höheres Problemverhalten im Umgang mit Gleichaltrigen zeigen, je inklusiver die Klassenkomposition hinsichtlich der Zusammensetzung von SchülerInnen mit und ohne SPF ist, unabhängig vom Anteil an anwesenden Kindern mit MIG (M10: b=0,22; $p<0,05)$. Auch bei simultanem Einbezug der sozioökonomischen und ethnischen Klassenkomposition ist festzuhalten, dass nicht die inklusive und auch nicht die ethnische Klassenkomposition mit dem Problemverhalten im Umgang mit Gleichaltrigen assoziiert ist, sondern die sozioökonomische Klassenkomposition (M11: $\mathrm{b}=0,07 ; p>0,05)$. Folglich kann Hypothese $3 \mathrm{~d}$ bestätigt werden, Hypothese $3 \mathrm{c}$ allerdings nicht.

\section{Fazit und Diskussion}

\subsection{Diskussion}

Ziel des Beitrags war es, zu überprüfen, ob der Besuch einer inklusiven Klasse mit dem sozialen Verhalten von SchülerInnen ohne SPF assoziiert ist. Bisherige Studien vermitteln diesbezüglich ein eher negatives Bild. Daher wurde mehrebenenanalytisch untersucht, ob der Besuch einer inklusiven Klasse unter Kontrolle individueller 
Merkmale und Merkmalen der Klassenkomposition mit dem prosozialen Verhalten und dem Problemverhalten im Umgang mit Gleichaltrigen zusammenhängt.

Entgegen der Annahmen zeigt sich aufgrund des Besuchs einer inklusiven Klasse kein genereller Unterschied im prosozialen Verhalten von SchülerInnen ohne SPF. Auch zwischen der inklusiven Klassenkomposition und dem prosozialen Verhalten von SchülerInnen ohne SPF sind keine Zusammenhänge auszumachen. Diese Befunde sind auch dann stabil, wenn weitere Merkmale der Klassenkomposition berücksichtigt werden. Folglich kann abgeleitet werden, dass SchülerInnen ohne SPF in inklusiven Klassen kein geringeres prosoziales Verhalten zeigen als ihre Peers in nicht-inklusiven Klassen, auch nicht bei einem steigenden Anteil an SchülerInnen mit SPF in den Klassen. Entsprechend ist auch nicht davon auszugehen, dass inklusive Settings hinsichtlich der Prosozialität ungünstige Peereffekte hin zu weniger sozialen Verhalten begünstigen. Die postulierten Befunde aus anderen Forschungsarbeiten (Gottfried 2014) lassen sich anhand dieser Daten für das prosoziale Verhalten also nicht replizieren. Dies ist möglicherweise darauf zurückzuführen, dass in den Klassen prozentual gesehen überwiegend SchülerInnen mit SPF im Bereich Lernen anwesend waren. Wenngleich auch dieser SchülerInnengruppe sozial ungünstiges Verhalten zugeschrieben wird, besteht ihr hauptsächlicher Förderbedarf im kognitiven Bereich. Möglicherweise sind diese Auffälligkeiten für SchülerInnen ohne SPF nicht ersichtlich, weshalb auch kein Angleichungsprozess stattfindet.

Hinsichtlich des Problemverhaltens im Umgang mit Gleichaltrigen und dem Besuch einer inklusiven Klasse besteht allerdings ein positiver Zusammenhang. SchülerInnen ohne SPF berichten von höherem Problemverhalten im Umgang mit Gleichaltrigen. Darüber hinaus ist erkennbar, dass das Problemverhalten im Umgang mit Gleichaltrigen steigt, je höher der Anteil an SchülerInnen mit SPF in den Klassen ist. Dies zeigt sich allerdings nicht mehr, sobald auch die sozioökonomische Klassenkomposition berücksichtigt wird. Stattdessen schätzen SchülerInnen ohne SPF ihr Problemverhalten im Umgang mit Gleichaltrigen höher ein, je höher der Anteil an SchülerInnen mit niedrigem sozioökonomischen Status ist, unabhängig davon wie hoch der Anteil an SchülerInnen mit SPF in den Klassen ist. Die ethnische Klassenkomposition hingegen kann nicht als Erklärung herangezogen werden. Allerdings vermittelt bereits der Vergleich der Stichprobengruppen der vorliegenden Analysen, dass sich in inklusiven Klassen ein deutlich höherer Anteil an SchülerInnen mit niedrigem SES befindet. Die Befunde gehen bspw. mit den Ergebnissen von Kellam et al. (1998) einher, dass gerade in Klassen mit einer höheren Armutsquote höherer Aggressionslevel herrscht. Auch dort ist nicht der individuelle Status erklärend für individuelles Verhalten, sondern erst die Wirkung des Merkmals auf Klassenebene.

Entsprechend geben die vorliegenden Ergebnisse Hinweise darauf, dass mit der Strukturierung von Klassen bereits negative Peereffekte begünstigt werden. Es ist nicht davon auszugehen, dass eine Klassenkomposition mit niedrigem sozioökonomischen Status ausschließlich über die SchülerInnen mit SPF vermittelt wird, auch wenn SchülerInnen mit SPF durchschnittlich betrachtet einen niedrigeren SES haben als ihre Peers ohne SPF (Henke et al. 2017). Die SchülerInnen mit SPF sind breit über die einbezogenen Klassen verteilt, wonach letztlich durchschnittlich 1,8\% SchülerInnen mit SPF die inklusiven Klassen besuchen. Folglich sollte es gerade bei 
der Bildung inklusiver Klassen vermieden werden, SchülerInnen mit SPF vermehrt in Klassen mit einem sozioökonomisch niedrigen Status zu platzieren, da in diesen Settings ohnehin schon mit einer erhöhten Wahrscheinlichkeit negative Peereffekte hinsichtlich sozialen Verhaltens stattfinden können.

Darüber hinaus deuten die vorliegenden Ergebnisse darauf hin, dass dem Schulkontext insbesondere in inklusiven Bildungssettings die wesentliche Aufgabe zukommt, zu berücksichtigen, dass soziale Verhaltensweisen in strukturierten und informellen Angeboten vermittelt und beeinflusst werden. Folglich sollten soziale Verhaltensweisen und Kompetenzen sowohl von SchülerInnen mit als auch ohne SPF insbesondere in inklusiven Lernumgebungen als auch mit einer Klassenkomposition mit niedrigem sozioökonomischer Status gezielt gefördert werden. Etwa durch Intervention der Lehrkraft, Aufklärung über sozial ungünstige Verhaltensweisen und wiederholtes Training mit Gruppen mit unterschiedlich ausgeprägten sozialen Verhaltensweisen können positive individuelle soziale Verhaltensweisen bestärkt werden (Kellam et al. 1998).

\subsection{Limitationen}

Die Ergebnisse der vorliegenden Untersuchung müssen insofern limitiert werden, als dass eine inklusive Klasse lediglich im Sinne eines Platzierungsmerkmals operationalisiert werden konnte. Der angesprochenen Relevanz, auch die Prozessebene von Unterricht zu berücksichtigen (Battistich et al. 1997; Kellam et al. 1998; Müller et al. 2018), konnte mit dem verwendeten Datensatz nicht Rechnung getragen werden. Es liegen zu wenige Informationen zur Gestaltung von Unterricht vor, insbesondere hinsichtlich der Organisation von inklusivem Unterricht oder zu Prozessen zwischen SchülerInnen mit und ohne SPF. So fehlen bspw. Angaben darüber, wie die SchülerInnen mit SPF in den Unterricht eingebunden sind, ob sie in einzelnen Fächern möglicherweise segregiert lernen und wie viel Zeit sie tatsächlich mit ihren Peers ohne SPF im Klassenverbund verbringen.

In die Stichprobe gingen durchschnittlich fünf Kinder pro Klasse ein. Da die Teilnahme an der NEPS-Erhebung freiwillig ist, ist es nicht auszuschließen, dass grundsätzlich eine positiv selektierte Stichprobe hinsichtlich des sozialen Verhaltens zustande kam. Dies kann allerdings nicht näher geprüft werden, da die Grundgesamtheit an SchülerInnen aufgrund der freiwilligen Teilnahme nicht identifiziert und das soziale Verhalten über alle SchülerInnen hinweg geprüft werden kann. Die Teilnahmequote lässt außerdem keine Aussage darüber zu, ob auf Klassenebene in inklusiven Klassen generell ein ungünstigeres soziales Verhalten besteht als in nichtinklusiven Klassen und inwiefern sich das aggregierte soziale Verhalten auf individuelles soziales Verhalten auswirkt. Das bedeutet auch, dass nur bedingt von einem vorliegenden Peereinfluss gesprochen werden kann, da hier rein mit der Platzierung von SchülerInnen in Klassen gearbeitet wurde und nicht etwa mit Merkmalen des Kontakts, Freundschaften o. ä.. Es konnte lediglich festgestellt werden, welche Art von Klasse die SchülerInnen für ein Schuljahr besuchten und welche Zusammenhänge zwischen dieser Erfahrung und der nachgelagert erhobenen abhängigen Variablen bestehen. Um von einem tatsächlichen Einfluss sprechen zu können, sollte einerseits eine Ausgangsmessung des SDQ erhoben werden und andererseits wiederholte Er- 
hebung in den folgenden Schuljahren stattfinden. Für zukünftige Studien ist daher zu empfehlen, das soziale Verhalten im Längsschnitt inklusive einer Ausgangsmessung $\mathrm{zu}$ modellieren.

Da an der NEPS-Erhebung designbedingt nur eine kleine Stichprobe an SchülerInnen mit SPF an Regelschulen teilnahm, von der der Großteil einen Förderbedarf im Bereich Lernen aufweist, konnten keine differenzierten Betrachtungen hinsichtlich des Aufkommens nach Förderschwerpunkten in den Klassen vorgenommen werden. Damit geht einher, dass nicht näher betrachtet werden konnte, ob etwa der negative Zusammenhang zwischen dem Besuch einer inklusiven Klasse und dem Problemverhalten im Umgang mit Gleichaltrigen auf die Anwesenheit von SchülerInnen mit bestimmten Förderbedarfen zurückzuführen ist und nicht wie hier angenommen ein genereller Effekt über alle Förderbereiche vorliegt. So konnte bspw. nicht untersucht werden, ob SchülerInnen mit SPF emotionale und soziale Entwicklung, denen am ehesten Verhaltensauffälligkeiten und ähnliches zugeschrieben werden, das soziale Verhalten ihrer Peers ohne SPF beeinflussen.

Funding Open Access funding enabled and organized by Projekt DEAL.

Open Access Dieser Artikel wird unter der Creative Commons Namensnennung 4.0 International Lizenz veröffentlicht, welche die Nutzung, Vervielfältigung, Bearbeitung, Verbreitung und Wiedergabe in jeglichem Medium und Format erlaubt, sofern Sie den/die ursprünglichen Autor(en) und die Quelle ordnungsgemäß nennen, einen Link zur Creative Commons Lizenz beifügen und angeben, ob Änderungen vorgenommen wurden.

Die in diesem Artikel enthaltenen Bilder und sonstiges Drittmaterial unterliegen ebenfalls der genannten Creative Commons Lizenz, sofern sich aus der Abbildungslegende nichts anderes ergibt. Sofern das betreffende Material nicht unter der genannten Creative Commons Lizenz steht und die betreffende Handlung nicht nach gesetzlichen Vorschriften erlaubt ist, ist für die oben aufgeführten Weiterverwendungen des Materials die Einwilligung des jeweiligen Rechteinhabers einzuholen.

Weitere Details zur Lizenz entnehmen Sie bitte der Lizenzinformation auf http://creativecommons.org/ licenses/by/4.0/deed.de.

\section{Literatur}

Battistich, V., Solomon, D., Watson, M., \& Schaps, E. (1997). Caring school communities. Educational Psychologist, 32(3), 137-151.

Bierhoff, H.-W. (2002). Prosocial behaviour (1. Aufl., Social psychology). East Sussex: Psychology Press, Routledge.

Blossfeld, H.-P., \& Roßbach, H.-G. (Hrsg.). (2011). Education as a Lifelong Process. The German National Educational Panel Study (NEPS) (Zeitschrift für Erziehungswissenschaft: Sonderheft 3, 2. überarbeitete Aufl.). Wiesbaden: Springer VS.

Demanet, J., \& van Houtte, M. (2011). Social-ethnic school composition and school misconduct. Does sense of futility clarify the picture? Sociological Spectrum, 31(2), 224-256. https://doi.org/10.1080/ 02732173.2011 .541343 .

Dishion, T. J., \& Tipsord, J. M. (2011). Peer contagion in child and adolescent social and emotional development. Annual review of psychology, 62, 189-214. https://doi.org/10.1146/annurev.psych.093008. 100412.

Fabes, R. A., Martin, C.L., \& Hanish, L.D. (2009). Children's behaviors and interactions with peers. In K. H. Rubin, W. M. Bukowski \& B.P. Laursen (Hrsg.), Handbook of peer interactions, relationships, and groups (Social, emotional, and personality development in context, S. 45-62). New York: Guilford. 
Fend, H. (2006). Neue Theorie der Schule. Einführung in das Verstehen von Bildungssystemen (1. Aufl.). Wiesbaden: VS Verlag für Sozialwissenschaften/GWV Fachverlage GmbH Wiesbaden.

Fischbach, A., Schuchardt, K., Mähler, C., \& Hasselhorn, M. (2010). Zeigen Kinder mit schulischen Minderleistungen sozio-emotionale Auffälligkeiten? Zeitschrift für Entwicklungspsychologie und Pädagogische Psychologie, 42(4), 201-210. https://doi.org/10.1026/0049-8637/a000025.

Frostad, P., \& Pijl, S. J. (2007). Does being friendly help in making friends? The relation between the social position and social skills of pupils with special needs in mainstream education. European Journal of Special Needs Education, 22(1), 15-30. https://doi.org/10.1080/08856250601082224.

Ganzeboom, H. B., \& Treiman, D. J. (1996). Internationally comparable measures of occupational status for the 1988 international standard classification of occupations. Social Science Research, 25, 201-239.

Ganzeboom, H. B., de Graaf, P. M., \& Treiman, D. J. (1992). A standard international socio-economic index of occupational status. Social Science Research, 21, 1-56.

Goodman, R. (1997). The strengths and difficulties questionnaire. A research note. The Journal of Child Psychology and Psychiatry, 38(5), 581-586.

Gottfried, M. A. (2014). Classmates with disabilities and students' noncognitive outcomes. Educational Evaluation and Policy Analysis, 36(1), 20-43. https://doi.org/10.3102/0162373713493130.

Grünke, M., \& Grosche, M. (2014). Lernbehinderung. In G. W. Lauth, M. Grünke \& J. C. Brunstein (Hrsg.), Interventionen bei Lernstörungen: Förderung, Training und Therapie in der Praxis (S. 76-89). Göttingen: Hogrefe.

Hartup, W. W. (2009). Critical issues and theoretical viewpoints. In K.H. Rubin, W. M. Bukowski \& B.P. Laursen (Hrsg.), Handbook of peer interactions, relationships, and groups (Social, emotional, and personality development in context, S. 3-19). New York: Guilford.

Hazzard, A. (1983). Children's experience with, knowledge of, and attitude toward disabled persons. The Journal of Special Education, 17(2), 131-139.

Henke, T., Bosse, S., Lambrecht, J., Jäntsch, C., Jaeuthe, J., \& Spörer, N. (2017). Mittendrin oder nur dabei? Zum Zusammenhang zwischen sonderpädagogischem Förderbedarf und sozialer Partizipation von Grundschülerinnen und Grundschülern. Zeitschrift für Pädagogische Psychologie, 31(2), 111-123. https://doi.org/10.1024/1010-0652/a000196.

Hennig, T., Schramm, S.A., \& Linderkamp, F. (2017). Einschätzung des Arbeits- und Sozialverhaltens durch Lehrkräfte - eine Validierungsstudie. Empirische Sonderpädagogik, 1, 52-65.

Hofmann, V., \& Müller, C. M. (2018). Avoiding antisocial behavior among adolescents: the positive influence of classmates' prosocial behavior. Journal of adolescence, 68, 136-145. https://doi.org/10.1016/ j.adolescence.2018.07.013.

Howes, C. (1988). Peer interaction of young children (Monographs of the Society for Research in Child Development, Bd. 53, with commentary by Kenneth H. Rubin and Hildy S. Ross and Doran C. French).

Hox, J.J. (2010). Multilevel analysis. Techniques and applications (2. Aufl., Quantitative methodology series). New York: Routledge.

Kanning, U. P. (2002). Soziale Kompetenz - Definition, Strukturen und Prozesse. Zeitschrift für Psychologie, 210(4), 154-163. https://doi.org/10.1026//0044-3409.210.4.154.

Kellam, S. G., Ling, X., Merisca, R., Brown, H.C., \& Ialongo, N. (1998). The effect of the level of aggression in the first grade classroom on the course and malleability of aggressive behavior into middle school. Development and Psychopathology, 10, 165-185.

Kiesner, J., Poulin, F., \& Nicotra, E. (2003). Peer relations across contexts: individual-network Homophily and network inclusion in and after school. Child Development, 74(5), 1328-1343.

Kuhn, H. P., \& Fischer, N. (2011). Zusammenhänge zwischen Schulnoten und problematischem Sozialverhalten in der Ganztagsschule. Entwickeln sich Ganztagsschüler/-innen besser? Zeitschrift für Erziehungswissenschaft, 14(3), 143-162. https://doi.org/10.1007/s11618-011-0232-1.

Lohbeck, A., Schultheiß, J., Petermann, F., \& Petermann, U. (2015). Die deutsche Selbstbeurteilungsversion des Strengths and Difficulties Questionnaire (SDQ-Deu-S). Diagnostica, 61(4), 222-235. https:// doi.org/10.1026/0012-1924/a000153.

Mahoney, J.L., Cairns, B. D., \& Farmer, T.W. (2003). Promoting interpersonal competence and educational success through extracurricular activity participation. Journal of Educational Psychology, 95(2), 409-418. https://doi.org/10.1037/0022-0663.95.2.409.

McNamara, B. C., \& Wentzel, K. R. (2006). Friend influence on prosocial behavior. The role of motivational factors and friendship characteristics. Developmental Psychology, 42(1), 153-163. https://doi.org/ 10.1037/0012-1649.42.1.153. 
Müller, C. M., Hofmann, V., Begert, T., \& Cillessen, A.H. (2018). Peer influence on disruptive classroom behavior depends on teachers' instructional practice. Journal of Applied Developmental Psychology, 56, 99-108. https://doi.org/10.1016/j.appdev.2018.04.001.

Müller, C.M., Hofmann, V., Fleischli, J., \& Studer, F. (2015). Effects of classroom composition on the development of antisocial behavior in lower secondary school. Journal of Research on Adolescence, 26(2), 345-359. https://doi.org/10.1111/jora.12195.

Preuss-Lausitz, U. (2005). Zur Dynamik der sozialen Beziehungen in der Schule. Das Verhältnis der „schwierigen“ Kinder zu den Gleichaltrigen. In U. Preuss-Lausitz (Hrsg.), Verhaltensauffällige Kinder integrieren. Zur Förderung der emotionalen und sozialen Entwicklung (S. 159-186). Weinheim: Beltz.

Ruijs, N. M., \& Peetsma, T.T. (2009). Effects of inclusion on students with and without special educational needs reviewed. Educational Research Review, 4(2), 67-79. https://doi.org/10.1016/j.edurev.2009.02. 002 .

Schwab, S., Gebhardt, M., Hessels, M. G. P., \& Nusser, L. (2016). Predicting a high rate of self-assessed and parent-assessed peer problems-Is it typical for students with disabilities? Research in developmental disabilities, 49-50, 196-204. https://doi.org/10.1016/j.ridd.2015.11.026.

Sekretariat der Ständigen Konferenz der Kultusminister der Länder in der Bundesrepublik Deutschland (2016). Sonderpädagogische Förderung in Schulen 2005 bis 2014 (Statistische Veröffentlichungen der Kultusministerkonferenz, Dokumentation Nr. 210). Berlin.

Stein, R., \& Müller, T. (2018). Verhaltensstörungen und emotional-soziale Entwicklung: zum Gegenstand. In R. Stein \& T. Müller (Hrsg.), Inklusion im Förderschwerpunkt emotionale und soziale Entwicklung (2. Aufl., Inklusion in Schule und Gesellschaft, Bd. 5, S. 22-47). Stuttgart: Kohlhammer.

Szczesny, M., \& Watermann, R. (2011). Differenzielle Einflüsse von Familie und Schulform auf Leseleistung und soziale Kompetenzen. Journal for Educational Research Online, 3(1), 168-193.

Tenorth, H.-E. (2013). Inklusion - Prämissen und Problemzonen eines kontroversen Themas. In J. Baumert, V. Masuhr, J. Möller, T. Riecke-Baulecke, H.-E. Tenorth \& R. Werning (Hrsg.), Inklusion. Forschungsergebnisse und Perspektiven (Schulmanagement-Handbuch, Bd. 146, S. 6-14). München: Oldenbourg.

Wentzel, K. R., \& Erdley, C. A. (1993). Strategies for making friends. Relations to social behavior and peer acceptance in early adolescence. Developmental Psychology, 29(5), 819-826.

Zander, L., Kreutzmann, M., \& Hannover, B. (2017). Peerbeziehungen im Klassenzimmer. Zeitschrift für Erziehungswissenschaft, 20(3), 353-386. https://doi.org/10.1007/s11618-017-0768-9. 